\title{
TECNOLOGÍAS DE INFORMACIÓN Y COMUNICACIÓN: EL CASO DE LAS COMUNIDADES INDÍGENAS CABÉCARES DE CHIRRIPÓ DE COSTA RICA
}

Cristian Brenes Granados'

Resumen

El presente trabajo acompaña a un producto de software titulado "Curso de lengua y cosmovisión cabécar", el cual hace un abordaje de la visión de mundo de las comunidades indígenas cabécares de Chirripó de Costa Rica a través de su lengua, mismo que marca la introducción de TICs como una oportunidad para construir puentes que permitan una convivencia intercultural y el reconocimiento del aporte de este grupo humano a la sociedad nacional.

Palabras claves: tecnología de la información, cultura indígena.

\section{Abstract}

This article presents a software called "Cosmo vision and mother tongue Cabecar Course", which presents the world vision thorough the mother tongue of Costa Rican Cabecares indigenous communities located in Chimipo. Also shows that information and communication technologies are an opportunity to build a relation that allows intercultural coexistence and recognize the contribution to Costa Rican society of this human group.

Keywords: informational technology, indigenous culture.

L

os pueblos indígenas se encuentran en condiciones de mayor desventaja que el resto de la población en todos los países de América Latina. En una reiterada negación, el tema se presenta con toda la carga histórica de exclusión de la población indígena y de su invalidación ciudadana. De esta

Universidad de Costa Rica, sedc del Atlántico, cbrenes@cariari.ucr.ac.cr Recibido: 4 de marzo-2007 - Aprobado: 30 de abril-2007 
manera, el nivel de subyugación que han padecido por siglos los pueblos indígenas parece estar asociado con su condición, que ha limitado las oportunidades de empleo, educación, salud, acceso a infraestructura adecuada y otros servicios básicos, y más aún con variables tales como el acceso a las Tecnologías de Información y Comunicación.

Uno de los mayores retos y desafios de la educación en poblaciones vulnerables es disminuir la brecha digital; que en el caso de los pueblos indígenas alcanza alarmantes proporciones para llevarlos a la clase de los informados, de los incluidos, permitiendo el desarrollo individual y colectivo.

\section{Las Tecnologías de Información y Comunicación (TICs) y su papel en la Sociedad de la Información y la Comunicación de las comunidades indígenas}

El término Sociedad de la Información y la Comunicación responde a un gran discurso ideológico. Primeramente, a mediados de la década de los 90, la Unión Europea se lanzó en un intento por regular y privatizar el sector de telecomunicaciones con el fin de permitirse competir con EE. UU. y Japón, en tecnologías de la información. El término fue escogido para subrayar que la nueva sociedad, por la cual ellos estaban luchando, tendría un importante eje social. De igual forma, podríamos citar variadas versiones, mismas que desafortunadamente han hecho inversiones mínimas en acciones orientadas al logro de los fines sociales.

Por lo anterior, no existe un concepto universalmente aceptado de lo que se le llama "Sociedad de la Información", aunque sí podemos precisar que alrededor de 1970 se inició un cambio en la manera en que las sociedades funcionan; este cambio se refiere básicamente a que los medios de generación de riqueza, poco a poco, se están trasladando de los sectores industriales a los sectores de servicios. En otras palabras, se supone que en las sociedades modernas, la mayor parte de los empleos ya no estarían asociados con las fábricas de productos tangibles, sino con la generación, almacenamiento y procesamiento de todo tipo de información, donde los sectores relacionados con las Tecnologías de Información y Comunicación (TICs), desempeñan un papel muy importante dentro de este esquema.

Para efectos de este artículo, partiremos de la siguiente reflexión para fundamentar un referente pertinente de la Sociedad de la Información y la Comunicación en función de nuestros propósitos, emanado de la Cumbre 
Mundial sobre la Sociedad de la Información, realizada en Caracas, en el mes de agosto de 2005:

"La nueva Sociedad de la Información deberá comprender un nuevo orden económico internacional, una mejor relación entre la humanidad y el planeta, incluido el respeto al medio ambiente y las políticas ecológicas y en general todo aquello que evite que el productivismo tecnocientífico continúe imponiendo su lógica destructiva e irresponsable para el futuro del planeta y de la especie humana. Solo en este sentido deberá plantearse la nueva sociedad de la información, aprovechando las nuevas tecnologías y adelantos científicos para expandir la defensa y la cultura de respeto a los Derechos Humanos".

Por lo anterior, defenderemos la posición que la Sociedad de la Información y la Comunicación es primero que nada una construcción social, que debe partir de la colectividad y que más que nunca, ha de contribuir a identificar un modelo que sea enteramente endógeno, de acuerdo con las tradiciones históricas, culturales y sociales, que considere las posibilidades y debilidades, pero que necesariamente debe estar a tono con la actual dinámica de crecimiento y oportunidades que ofrece el mundo moderno, de forma de garantizar el acceso de todos a esta construcción (Rodríguez, 2004, p. 5).

Es en este concepto de Sociedad de la Información y la Comunicación en el que las comunidades indígenas tienen cabida, y se hace necesario indicar que en este contexto, las Tecnologías de Información y Comunicación (TICs) son vistas como el conjunto de herramientas a través de las cuales se hace posible la concreción de tal fin.

Es necesario tener en cuenta que al introducir tecnologías de la información y la comunicación en una comunidad indígena, llegan junto a ellas los medios de información, la cultura popular y los idiomas mundiales como el inglés, que provocan situaciones de multiculturalidad ineludibles con las tradiciones locales, creando divergencias respecto de las formas de percibir el mundo.

La razón de este artículo es reflejar a través del producto desarrollado, cómo estas tecnologías, en este mar de contradicciones, pueden representar, para una comunidad indígena, una herramienta para difundir, preservar, fomentar y fortalecer su propia lengua y cultura. 


\section{El impacto de la colonización en la distribución de los asentamientos indígenas de Costa Rica}

Apoyado en investigaciones históricas (Ibarra, 1990), es posible reconstruir un panorama de la ocupación territorial a la llegada de los europeos y la identidad de los pueblos que la protagonizaron.

Cabe señalar que los españoles protagonizaron desplazamientos forzados de poblaciones enteras hasta por lo menos el siglo XVIII, con el fin de repoblar regiones bajo su dominio en que la población fue exterminada por las enfermedades o por el rẻgimen de explotación instaurado. Este fue el caso de la actual región del Valle Central en que se asentaron los españoles con más ahínco. La población indígena presente en esta región, y conocida en los documentos históricos como Huetar, fue prácticamente exterminada entre los siglos XVI y XVIII.

En otra zona del país, la de Guanacaste hacia el noroeste, se dio un proceso de etnocidio y genocidio parecido al de la región central. Los chorotegas, y otros pueblos reseñados por los documentos históricos, fueron aniquilados física y culturalmente. Los borucas son el único pueblo situado en la región sur de Costa Rica que lograron sobrevivir a la conquista y a la colonia, conservando su identidad cultural, a pesar de los efectos de la dominación española. Sin embargo, han sufrido un proceso intenso de aculturación en los últimos años.

La región atlántica del país presentaba una situación bastante diferente a la del Pacífico. Esta región, tanto por sus características físicas (topografía quebrada, selva tropical húmeda y densa) como por la tenaz resistencia de sus pobladores originales, desestimuló muchos de los intentos de conquista emprendidos por los españoles. Situación que les ha permitido ir a la vanguardia tanto en territorio ocupado como en habitantes por grupo étnico y extensivamente a sus posibilidades de permanecer en condición de arraigo cultural (Mora, 2002, p. 27).

Para ese momento, estos pueblos utilizaban Chirripó como refugio para la preservación y continuación de su cultura; los procesos civilizatorios, venidos desde europeos y mestizos durante siglos, encontraron en Chirripó una zona de dificil acceso, por lo que se convirtió en una barrera para la visión desarrollista del Valle Central.

A pesar de que no podemos relacionar completamente los cabécares con ninguna tradición arqueológica, las correspondencias etnográficas como las comentadas por Stone (1952) nos sirven de base para suponer que, pese a 
las diferentes migraciones y procesos de mestizaje sufridos por estos grupos en los últimos 500 años, es posible hablar de una conexión entre la evidencia arqueológica de la zona atlántica con los actuales grupos indígenas costarricenses asentados en la misma región. Esto les confiere una profunda dimensión histórica a estas comunidades.

Por tal razón, reconocer la larga tradición cultural de estos pueblos, es reconocer su amplio trayecto histúrico, y su derecho impostergable de ser tomados en cuenta en toda acción política que se proponga en sus territorios.

En el siguiente mapa se representa el posicionamiento territorial de los actuales grupos indígenas costarricenses; se nota que de la colonización a la actualidad, los grupos indígenas que han sobrevivido a este proceso han antepuesto el principio de equilibrio y convivencia con la naturaleza como un mecanismo que les garantiza la preservación de su identidad cultural.

\section{Mapa 1}

Mapa de grupos indígenas en Costa Rica con las regiones geográficas ocupadas.

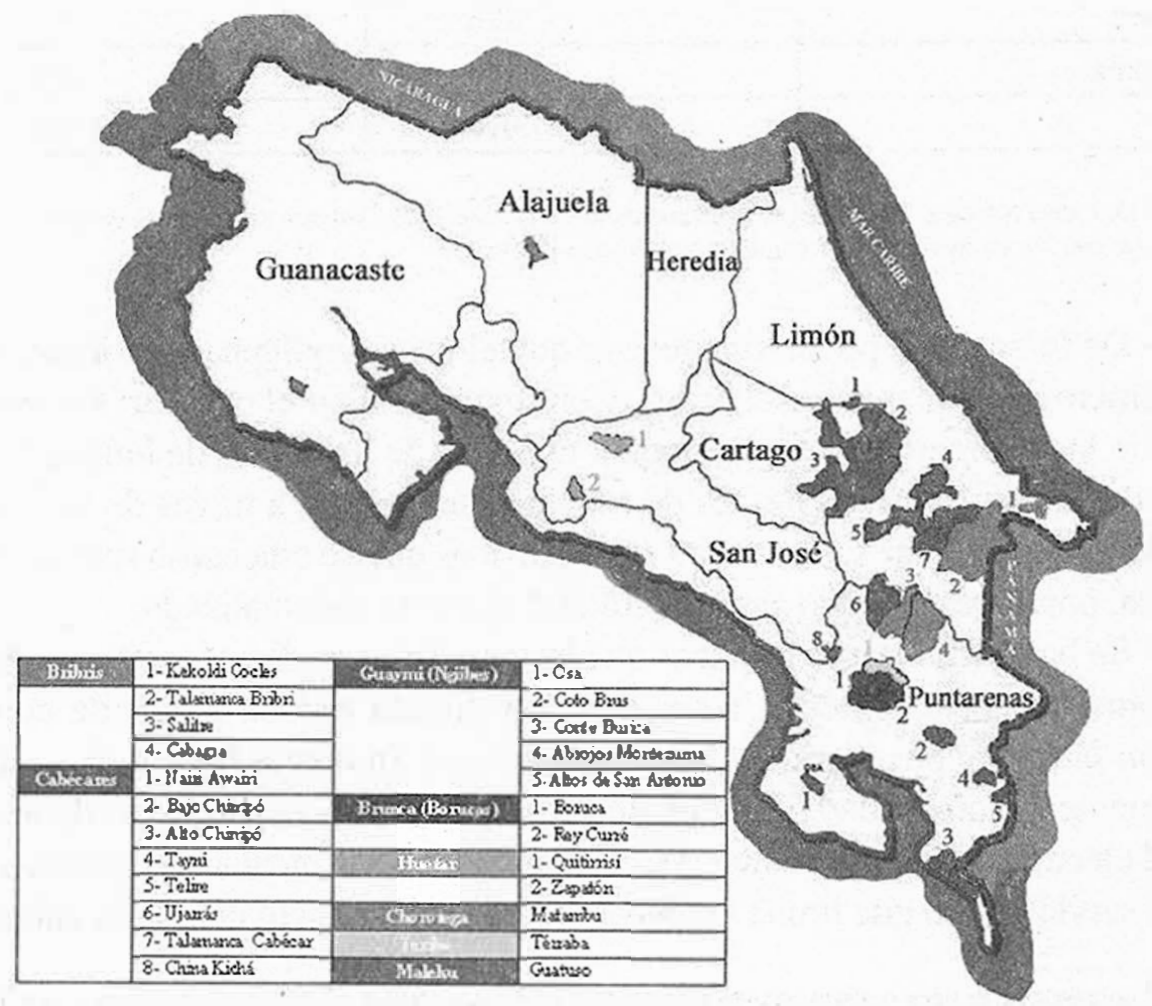

Fuente: http://www.mcjdcr.go.cr/Territorio\%20Indigena/index.htm Recditado por Cristian Brenes G. 
Tal y como se muestra, los dos grupos mayoritarios en ocupación territorial son los cabécares y los bribris, característica que persiste en el porcentaje de hablantes de lengua nativa por grupo indígena (véase el Cuadro 1).

\section{Cuadro 1}

Cantidad de personas por grupo indígena y hablantes nativos.

\begin{tabular}{|l|c|c|}
\hline \multicolumn{1}{|c|}{ Grupo indigena } & $\begin{array}{c}\text { Número de personas que } \\
\text { pertenece al grupo }\end{array}$ & $\begin{array}{c}\text { Personas hablantes del } \\
\text { idioma nativo }\end{array}$ \\
\hline Bribris & 9.839 & 5.647 \\
\hline Cabécares & $\begin{array}{r}9.679 \\
\text { 4.619 personas de } \\
\text { Alto Chirripó }\end{array}$ & 7.622 \\
\hline Guaynníes (Ngöbes) & 2.487 & 2.041 \\
\hline Bruncas (Bonucas) & 1.856 & 70 \\
\hline Huetares & 1.002 & 7 \\
\hline Chorotegas & 991 & 16 \\
\hline Teribes & 726 & 57 \\
\hline Malekus & 436 & 300 \\
\hline & 27.016 & 15.760 \\
\hline
\end{tabular}

Fuente: IX Censo Nacional de Población realizado en junio del año 2000, Sistema de consultas en Linea REDATAM, http://www.inec.go.cr/. Recopilado por Cristian Brenes G.

De lo anterior, podemos precisar que el grupo indígena más importante en número de habitantes es el bribri, y en segundo lugar el cabécar; sin embargo, son los cabécares quienes ostentan el índice de hablantes de lengua nativa más alto. Ante esta situación, es de esperar que puedan, a través de la lengua, mantener y preservar su identidad cultural. Y es que se cita como referencia la lengua, porque es el único aspecto cultural que está sistematizado.

Se hace importante destacar que las condiciones de acceso tan difíciles de estos territorios, pueden tener relación directa con su grado de conservación cultural, por ejemplo: en Alto Chirripó (nuestro objeto de estudio) solamente la comunidad indígena de Kabebata cuenta con camino de acceso y red electrificada; las restantes 58 comunidades identificadas no cuentan con estos servicios $^{2}$, lo que limita las posibilidades de contacto con otras culturas.

2 Actualmente no se tiene registro preciso de la cantidad de comunidades existentes en el territorio indigena de Alto Chirripó, sin embargo, las 59 referidas en este articulo corresponden a las localidades donde se ha establecido un centro escolar. 


\section{Los cabécares de Alto Chirripó}

La etnia cabécar se ha relacionado dentro de los grupos chibchoides del área intermedia de América, similar a los ngömes, guaymíes, bribris y cunas (Barrantes, 1998, p. 16). Los cabécares han estado durante siglos relacionados con los bribris y antes de la llegada de los españoles, los bribris habían conquistado a los cabécares mediante varios enfrentamientos, pero en lugar de desarrollarse una relación de vencido contra vencedor se creó a partir de ahí un patrón cultural de mutua dependencia, donde los bribris tenían la cabeza política de la comunidad y los segundos aportaban a los líderes espirituales. Ambos estaban organizados en clanes matriliniales y las prácticas funerarias les eran comunes realizando enterramientos secundarios y ritos específicos con ofrendas y cultos al espíritu del muerto (Stone, 1952, p. 3).

\section{Características geográficas del territorio de Alto Chirripó}

Los territorios indígenas asentados en la cuenca del río Chirripó se ubican en la zona climática denominada como bosque subtropical muy húmedo, con una precipitación anual entre los 2000 y $8000 \mathrm{~mm}$, y una temperatura promedio de 16 a 26 grados, la altitud va de 1000 a $2800 \mathrm{msnm}$, los asentamientos están sobre todo ubicados en los diferentes aluviones alrededor del Talweg que marca el curso del río sobre las estribaciones de la Fila Matama (Bozzoli, 1986, p. 23).

La topografia irregular ha obligado a estas poblaciones a mantener técnicas agrícolas de rotación de cultivos. Igualmente, por el relieve, las vías de comunicación terrestres existentes son difíciles para el acceso de vehículos y dependiendo de las condiciones climáticas, pueden resultar no aptas para medios de transporte animal.

En este marco, se hace cada vez más evidente la necesidad de la incorporación de las nuevas Tecnologías de Información y Comunicación como agente generador de alternativas de desarrollo para las comunidades indígenas, así como una oportunidad para el país en términos de reconocimiento y aceptación de las diferencias culturales aportadas por los diversos grupos indígenas.

\section{El escenario de las TICs en las comunidades indígenas cabécares de Alto Chirripó}

El territorio ocupado por los cabécares de Alto Chirripó posee condiciones de infraestructura y servicios verdaderamente diferentes al del resto de los grupos indigenas de Costa Rica. 
Como ya se mencionó, solamente una comunidad tiene acceso a través de camino para vehículos de doble tracción y es la única que cuenta con red de electricidad. De las restantes 58 comunidades podemos decir que las más cercanas están a hora y media a pie por entre la montaña y al aumentar las distancias, las limitaciones de acceso son más dificiles como: cruces de ríos de caudal considerable, veredas y trochas donde el barro alcanza profundidades de hasta $70 \mathrm{~cm}$, alta precipitación y topografia muy quebrada, entre otras.

Seguidamente, se presenta una descripción de las condiciones actuales para la introducción de nuevas Tecnologías de Información y Comunicación: el caso del computador.

\section{Energía eléctrica}

El Instituto Costarricense de Electricidad ${ }^{3}$ ha desarrollado un programa de paneles fotovoltaicos o paneles solares en el territorio indigena cabécar de Chirripó. La idea de la implementación de esta tecnología es "brindar un servicio limitado pero que sufraga las necesidades básicas de familias que durante decenios no contaban con electricidad y que no podian soñar con ver televisión, escuchar radio o tan siquiera tener iluminación nocturna"4. La cobertura de este servicio alcanza alrededor del $2 \%$ del total de la población; para el caso de las escuelas, de las 59 existentes en el territorio, 19 cuentan con panel. Cada sistema consta de un panel solar de 75 watts, un controlador, un inversor para producir corriente alterna, los disyuntores, una batería de ciclo profundo que permite el uso del sistema de 5:30 de la tarde a 9:00 de la noche, los cables eléctricos y apagadores necesarios, así como tres fluorescentes compactos de 15 watts cada uno. Con la descripción anterior, es claro que la idea no estaba asociada con el soporte a nuevas tecnologías como computadoras, ni equipos de telecomunicaciones. Sin embargo, se hace necesario indicar que, según pruebas realizadas por el autor en la escuela de Jameikiäri, un panel tiene la capacidad de mantener encendido un computador Pentium III, 256 RAM, 20 GB disco duro, monitor de 15" en una condición de día soleado, en ausencia de sol, es decir, con la batería previamente cargada, un panel solamente soporta 49 minutos.

3 Institución gubernamental que ofrece en Costa Rica los servicios de electricidad y telecomunicaciones.

4 Http://www.grupoice.com/esp/ele/infobase/pan_sol.htm 


\section{Equipo}

En estas condiciones, es difícil imaginar siquiera la existencia de la tecnología de las computadoras en este territorio; de hecho de las 59 escuelas $^{5}$ que se encuentran en el territorio, no existe una sola que tenga o haya tenido computadora. Para el caso de la Telesecundaria ${ }^{6}$ de Tsinikläri, el Profesor Regular posee un computador de escritorio de carácter personal. Para el caso de la comunidad en general, no existe una sola persona que cuente con una computadora.

\section{Telecomunicaciones}

La infraestructura de telecomunicaciones es igualmente deficiente, de hecho el único medio posible hasta el momento es la tecnología celular, aun considerando que tiene una tasa de cobertura cercana al 1\% del territorio. El caso de la radiofrecuencia es igualmente difícil de considerar debido a las condiciones de irregularidad del relieve. De hecho, por el momento, la tecnología en la que se puede pensar es la satelital. Sin embargo, el tema de las telecomunicaciones no está dentro de las prioridades de corto plazo debido a la ausencia de computadores en el territorio.

\section{La estrategia de aplicación de TIC en las comunidades indígenas Chirri- pó Cabécar}

Analizada la necesidad de la participación de las comunidades indígenas en la Sociedad de la Información y la Comunicación, sumada a las condiciones para las TICs en el territorio indígena cabécar de Alto Chirripó, y atendiendo lo estipulado en el artículo 16 del Proyecto de Declaración de los Derechos de los Pueblos Indígenas por parte de la ONU que establece que "los pueblos indígenas tienen derecho a que la dignidad y diversidad de sus culturas, historias, tradiciones y aspiraciones queden debidamente reflejadas en todas las formas de educación y en la información pública", se ha elaborado una propuesta de introducción de TICs que le permita al resto de la comunidad nacional acceder al conocimiento de la cultura cabécar y favorecer relaciones de valoración y respeto, y para las comunidades indígenas la posibilidad de mirar en las TICs una oportunidad de ser incluidas en este paradigma.

5 La primera escuela en el territorio indigena de Alto de Chirripó se fundó en 1986.

6 Es la única opción a nivel de secundaria en cl territorio indigena de Alto Chirripó, se fundó en el 2005. 
La propuesta construida se fundamenta en la necesidad no indígena del conocimiento de la comunidad cabécar desde su cosmovisión, es decir, que a través de una herramienta se pueda conocer y aprender: su historia, actividades agrícolas, conocimientos científico-culturales, visión de mundo, la espiritualidad, formas de vida, relaciones con su entorno natural, estructura de organización familiar y social, entre otros, fundamentada desde el aprendizaje de la lengua cabécar.

\section{Propuesta metodológica}

La construcción de este software pasó por cinco momentos, a saber:

\section{Acceso al contexto}

Aproximadamente hace tres años, cuando se realizó la primera gira al territorio de Alto Chirripó, a la comunidad de Tsipirí, no se tenía como objetivo la producción de un software multimedia, sino que el propósito era hacer un bordaje exploratorio del contexto, que le permitiera a la Universidad asumir una posición epistemológica, para darle pertinencia a las acciones por desarrollar en esta región. El equipo de trabajo interdisciplinario trata de cumplir este objetivo, y en esta incursión exploratoria de los territorios se empiezan a dibujar escenarios para que cada especialista pudiera aportar a esta vinculación. Después de una decena de giras a la región, se construyó en forma conjunta con las comunidades, una matriz de acciones dentro de las cuales la Universidad tomaría participación en algunas y, en otras, por su naturaleza, las referiría a otras instituciones, pues no se debía ni se deseaba asumir las responsabilidades estatales.

La matriz obedecía a un objetivo claramente trazado, que era: "Facilitar acciones educativas que promuevan la participación activa de los miembros de la comunidad cabécar de Chirripó en la construcción (búsqueda) de nuevas oportunidades de desarrollo". Dentro de esta matriz de acciones, se cubrieron los siguientes temas:

- Desarrollo de estética y creatividad.

- Identidad y rescate de la lengua cabécar.

- Potenciar prácticas agrícolas.

- Consolidación de redes de comercialización de productos.

- Formación de líderes comunales.

- Cursos de cosmovisión para la comunidad no indígena. 
- Introducción de TICs.

- $\quad$ Propuesta de una carrera interuniversitaria para formar maestros nativos con pertinencia cultural, titulada Bachillerato en educación en I y II ciclos con énfasis en educación intercultural bilingüe.

La realización de las giras no terminaron al concretar esa matriz; por el contrario, aquella primera etapa de contacto con las comunidades indígenas clarificó los ámbitos susceptibles de desarrollar desde el quehacer universitario. En el caso del área de Informática, la primera idea que se planteó fue el desarrollo de un mapa temático de la región, debido a que no existe una referencia de mapa o algún otro documento que precise las comunidades ni su ubicación geográfica. Para ello, se empezó a recorrer el territorio (siempre en compañía de nativos) y tomando como punto de referencia las escuelas, ya que su patrón de asentamiento es sumamente disperso y no tienen centros de población. Hasta la fecha, se han georreferenciado un total de 27 comunidades, considerando que para llegar a algunas de ellas, es necesario caminar de uno a dos días, tan sólo de ida (véase el Mapa 2).

\section{Mapa 2}

Ubicación geográfica de las comunidades indígenas en el territorio indígena de Alto Chirripó.

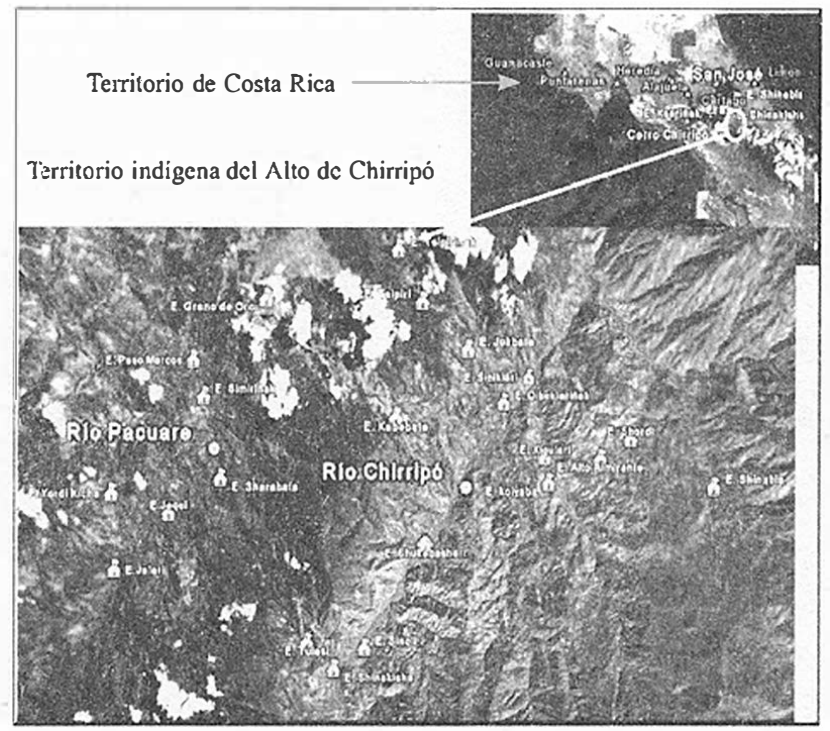

Fuente: Elaborado por el autor con datos referenciados y sobre la Plataforma Google Earth 2005 Freeware. 
Estas giras dieron la oportunidad de compartir con representantes de las comunidades desde distintos momentos de su cotidianidad y acercarse, poco a poco, al conocimiento de su cosmovisión y a una caracterización sociocultural de este grupo indígena. Aquí se realizaron registros digitales como fotografias y vídeos, así como entrevistas a informantes claves.

\section{Definición del guión}

Luego de analizar los insumos recogidos en las visitas a las comunidades, se estructuró el guión del software y se determinó que la oportunidad de desarrollo de las TICs, giraba en tomo a un proceso de visibilización y de reconocimiento, de tal forma que constituyera una oportunidad para que la población no indígena se acercara a la diversidad cultural de estas comunidades.

A partir de la caracterización sociocultural, se determinó la siguiente temática desarrollada y articulada en un menú tal y como lo muestra la Imagen 1: el clima, los alimentos y los animales cabécares, el tiempo, la casa, los personajes de la comunidad, la familia, la naturaleza y el cuerpo humano.

\section{Imagen 1}

Portada del Software Multimedia: Curso de Lengua y Cosmovisión Cabécar.

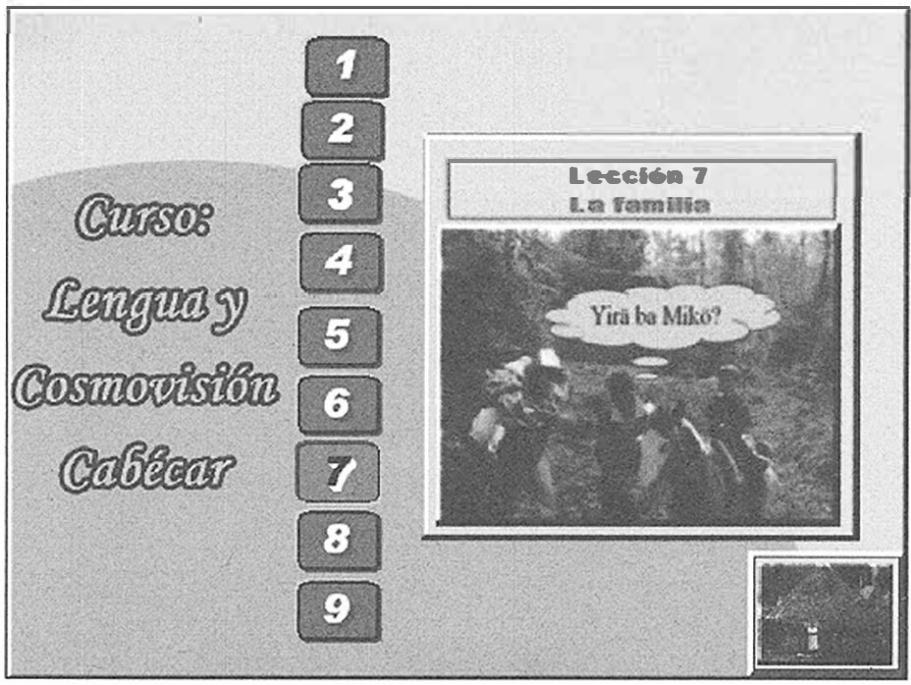

Fuente: Cristian Brenes G. 
Una vez seleccionados los temas por cubrir, se procedió a definir el eje temático que permitiría desarrollar el software; para ello, se tenían dos posibilidades: la lengua o la cultura. Aplicando el principio de que la lengua es un rasgo de la cultura, la decisión fue hacer un curso de Aprendizaje de Lengua Cabécar.

Seguidamente, se debía determinar la forma en la que se iba a abordar el tema. Por tratarse de un curso para el aprendizaje de un idioma, se procedió a la investigación tanto en Institutos (Centro Cultural Norteamericano), Universidades (Editorial UNED), Cursos Comerciales (New Horizonts, Interchange), Ministerio de Educación Pública, productos Paquetes Multimedia Comerciales, entre otros, con el fin de determinar un modelo metodológico apropiado. Al final se obtuvo la siguiente estructura: en total, se elaboraron 9 lecciones estructuradas cada una en 7 secciones, Objetivos, Vocabulario, Diálogo, Gramática, Práctica Escrita, Práctica Oral y Evaluación.

Además, cada lección tiene incluidos otros contenidos como: el abecedario, los pronombres, los clanes, saludos y frases comunes, contraposiciones, tiempos verbales, entre otros, que permiten enriquecer la experiencia de conocimiento de la cultura cabécar.

Un eje transversal en la construcción de este medio fue la pertinencia cultural o contextual y desde el mismo, se logró estructurar cada guión, escenario y personaje que evidencian las características identitarias de este grupo de población.

\section{Desarrollo de contenido}

Una vez obtenidos los temas, el paso siguiente fue desarrollar el contenido de cada tema. Para ello, se contó con la participación de personal docente del Programa de Lengua y Cultura del Ministerio de Educación Pública, destacado en el territorio de Alto Chirripó. La secuencia de construcción fue la siguiente: determinar los objetivos de aprendizaje de la lección e introducir un par de clips de vídeo y algunas imágenes que dieran una idea al usuario del contenido a tratar. Seguidamente, era necesario cubrir en forma selectiva, el vocabulario referente al tema, tarea que debía ser estrictamente realizada por los nativos de correspondencia con el conocimiento de la lengua.

La siguiente tarea implicaba la formulación de un diálogo que permitiera al usuario interactuar con el contenido, de forma que posibilitara la aplicación del vocabulario en la cotidianidad comunicativa; que al igual que el caso anterior no podía ser modelada por hablantes de español, ya que muchas 
de las palabras no tienen equivalente en el idioma cabécar. Ahora bien, se hacía necesario explicar la estructura del vocabulario en el contexto del diálogo presentada en la sección de gramática.

De esta sección en adelante, el software permite una mayor interacción y el usuario toma un papel más activo, ya que tiene que empezar a demostrar los conocimientos adquiridos por medio de dos prácticas de carácter formativo. La primera es escrita, donde se requiere de la memoria visual y la segunda es una práctica oral, donde el usuario aplica su memoria auditiva. Para finalizar, se realiza una evaluación que le permite al usuario tener una idea del grado de aprovechamiento de la lección.

\section{Recolección de insumos multimedios}

Una vez obtenido el guión gramatical, se procede a seleccionar los materiales digitales recolectados en la etapa de acceso al contexto, con el propósito de diseñar las interfaces multimedias. En el caso de los escenarios, fotografias, personajes, sonido ambiente son todos contextualizados, y las narraciones de los diálogos, palabras de vocabulario, oraciones se grabaron y luego se editaron una por una.

\section{Programación funcional}

Seleccionados todos los insumos, se procedió a la programación funcional. El grueso de esta recae en las secciones prácticas y la evaluación. El principio del Curso de Lengua evidenciaba la necesidad de que el usuario pudiera medir, de alguna manera, el nivel de aprendizaje obtenido, y ello obliga a dos cosas: la primera ofrecer una herramienta que le represente algún reto y segunda, el sentimiento de que está siendo evaluado. Esas dos consideraciones representan un reto importantísimo para el programador, que le da un valor agregado al producto desde el nivel de aprovechamiento del usuario.

\section{Conclusiones}

1. Los cabécares de Alto Chirripó pertenecen al grupo que no ha podido disminuir la brecha digital, y muy conscientes de los beneficios potenciales, así como de los retos y riesgos, sólo miran cómo las Tecnologías de Información y Comunicación constituyen un medio más de exclusión. 
2. Es necesario tener en cuenta que al introducir tecnologías de la información y la comunicación en una comunidad indígena, llegan junto a ellas los medios de información, la cultura popular y los idiomas mundiales como el inglés, que provocan tensiones ineludibles entre los aspectos identitarios locales y globales que se evidencian en la forma de percibir y entender el mundo. El producto aquí mostrado es una experiencia del uso de estas tecnologías, y como en este mar de contradicciones, puede representar para una comunidad indígena, una herramienta para difundir, preservar, fomentar y fortalecer su propia lengua y cultura.

3. Hace falta implementar iniciativas de introducción de las TICs, simultáneamente al desarrollo de una estrategia de capacitación de su uso, que considere que ellas no pueden sustituir, sino más bien complementar las formas tradicionales de expresión y de comunicación de las comunidades indígenas, en las cuales su cultura nace y vive de expresiones y representaciones orales.

4. No se debe seguir postergando la introducción de las TICs amparados en la carencia de la plataforma tecnológica en estas comunidades; por el contrario, se deben generar estrategias de encuentro que les permitan acceder a ellas para enriquecer los contactos y las vinculaciones desde ellos hacia los otros grupos humanos; de esta forma, van construyendo un referente del uso de la tecnología.

5. Se hace indispensable partir del principio de realidad que el participar de la Sociedad de la Información y la Comunicación, no implica cambios sustanciales en las condiciones de desigualdad, sino que es necesario el desarrollo paralelo de políticas de Estado y otras instancias que incidan en la disminución de las condiciones de exclusión social.

6. El software ofrece una posibilidad de sensibilización para las comunidades no indígenas que les permita construir mecanismos, valores y comportamientos que posibiliten una convivencia intercultural.

7. Uno de los mayores retos en este proceso de introducción de nuevas Tecnologías de Información y Comunicación en las comunidades indígenas de Alto Chirripó, es lograr que los depositarios del conocimiento ancestral (los mayores) mantengan su rol en el desarrollo de la organización política y social de la comunidad. 


\section{REFERENCIAS}

Barrantes, R. (1998). Origen y relaciones entre los Amerindios Chibcha de Costa Rica: una perspectiva genética y evolutiva, p. 57. Primer congreso científico sobre pueblos indígenas de Costa Rica y sus fronteras, compiladoras: Maria Eugenia Bozzoli, Ramiro Barrantes, Dinorah Obando y Mirna Rojas: UNICEF, UNED, UCR.

Bozzoli de Wille, M. E. (1986). El indigena costarricense y su ambiente natural: usos y adaptaciones. San José, Costa Rica: Editorial Porvenir.

Ibarra, E. (1990). Las sociedades cacicales de Costa Rica. San José: Editorial de la Universidad de Costa Rica.

Mora, J. (2002). Proyecto de un nuevo Estatuto Indigena para Costa Rica: Experiencias y Desafios. San José, OIT-Costa Rica.

Rodríguez, G. (2004). "Gobierno Electrónico: Hacia la Modernización y Transferencia de la Gestión Pública". Revista de Derecho (21), 1-23.

Stone, D. (1952). Living Archeology of the Bribri and Cabecar Indians of Costa Rica. Ponencia presentada en el 29 Congreso de Americanistas, Chicago. 